\title{
Balance analítico de la lectura y la escritura en educación básica
}

Modesta Capulín-Ballina

ballinamonse2016@gmail.com

Tesista de la Maestría en Investigación de la Educación

Instituto Superior de Ciencias de la Educación del Estado de México, México.

https://orcid.org/0000-0002-9452-2218

Recepción:15 de junio del 2020

Aprobación:07 de septiembre del 2020

Publicación: 06 de junio del 2021

\section{Resumen}

El presente artículo es un balance analítico de investigaciones que tiene que ver con la lectura y la escritura en el contexto escolar, para lo cual el objetivo fue identificar y analizar investigaciones relacionadas con la lectura y la escritura de los alumnos de educación básica. De ahí que, el método empleado fue cualitativo con modalidad no interactiva, con un proceso de cuatro etapas; la primera correspondió a la búsqueda de trabajos entre el 2000 y 2018, que describieron la lectura y la escritura, las prácticas sociales y la literacidad, en el contexto escolar. Los repositorios de indagación fueron revistas especializadas como, Revista Iberoamericana de Educación, Revista Mexicana de Investigación Educativa, Perfiles Educativos, además, tesis de maestría. La segunda etapa fue, seleccionar las investigaciones tomando en cuenta la estructura, es decir, que tuvieran introducción, metodología, resultados o hallazgos y conclusiones. En la tercera etapa se agruparon las investigaciones, tratando que guardaran relación entre sí, ya sea por el objeto de estudio, por sujetos de investigación o por el contexto (escolar o sociocultural); siendo evidente que la lectura y la escritura pocas veces fue estudiada de manera simultánea. Finalmente, en la cuarta etapa, se extrajo la información esencial para ir elaborando el documento. A saber, el análisis de las investigaciones presentadas, permitieron reconocer que en las últimas dos décadas han generado diversos estudios acerca la lectura y la escritura, que existen experiencias, proyectos, materiales educativos y diferentes metodologías para su enseñanza, a la vez que los actores educativos siguen con la idea que la lectura y la escritura son codificar o decodificar letras, mismas que tienen que alcanzar los alumnos en los primeros años de escolaridad. En este sentido, las conclusiones permiten tener un balance de los documentos citados, a la vez ponen sobre la mesa hacia dónde dirigir próximas investigaciones.

Palabras claves: Lectura, escritura, prácticas sociales, educación básica. 


\title{
Analytical balance of reading and writing in basic education
}

\begin{abstract}
This article is an analytical balance of research that has to do with reading and writing in the school context, for which the objective was to identify and analyze research related to reading and writing of basic education students. Hence, the method used was qualitative with a non-interactive modality, with a four-stage process; the first corresponded to the search for jobs between 2000 and 2018, which described reading and writing, social practices and literacy, in the school context. The research repositories were specialized journals such as, Revista Iberoamericana de Educación, Revista Mexicana de Investigación Educativa, Profiles Educativos, in addition, master's thesis. The second stage was to select the investigations taking into account the structure, that is, that they had an introduction, methodology, results or findings and conclusions. In the third stage, the investigations were grouped, trying to be related to each other, either by the object of study, by research subjects or by the context (school or sociocultural); being evident that reading and writing were rarely studied simultaneously. Finally, in the fourth stage, the essential information was extracted to prepare the document. Namely, the analysis of the research presented allowed us to recognize that in the last two decades they have generated various studies about reading and writing, that there are experiences, projects, educational materials and different methodologies for their teaching, at the same time that the actors Educational programs continue with the idea that reading and writing are to encode or decode letters, the same that students have to achieve in the first years of schooling. In this sense, the conclusions allow to have a balance of the cited documents, at the same time they put on the table where to direct future research.
\end{abstract}

Keywords: Reading, writing, social practices, basic education

\section{Introducción}

Hoy en día, la educación básica tiene varios retos, el principal sigue siendo, que los alumnos desarrollen habilidades comunicativas como, leer, escribir, hablar y escuchar. Sin embargo, la realidad en el contexto escolar vislumbra problemáticas relacionadas al proceso de la lectura y la escritura durante toda la escolaridad, en este tenor, algunos alumnos no alcanzan el perfil establecido en los Programas de Estudio vigentes, derivado que la lectura y la escritura la siguen entendiendo como habilidades para descifrar letras. 
De ahí la importancia de conocer, ¿cómo es la lectura y la escritura de los alumnos de educación básica?, ¿desde dónde se ha mirado la lectura y la escritura en los últimos tiempos?, ¿cómo se presentan en los diferentes contextos? y ¿cuál es el uso y el papel que representa la lectura y la escritura para los actores educativos? En consecuencia, para dar respuesta a dichas interrogantes y de forma paralela abrir nuevas líneas de investigación, se planteó como objetivo, identificar y analizar investigaciones relacionadas con la lectura y la escritura de los alumnos de educación básica, mientras que la pregunta orientadora fue, a partir de las investigaciones realizadas en las dos últimas décadas ¿cómo ha sido el proceso de la lectura y la escritura de los alumnos de educación básica?

En consecuencia, el objeto de estudio fue la lectura y la escritura, las cuales tienen que ver con situaciones de interacción, comunicación y reconocimiento, en un espacio, tiempo y circunstancia. En este sentido, son mucho más que el descifrado de letras, ya que están determinadas por las prácticas sociales, mismas que, según Zavala (2009) son la "manera de usar la lectura y la escritura en el marco de un propósito social específico" (p. 23). Por lo tanto, no solo se encuentran en contexto escolar, sino en los otros contextos donde el alumno interactúa.

Con respecto al método empleado, este fue cualitativo, con modalidad de investigación no interactiva, analítica, en la cual se "estudian conceptos y sucesos históricos a través de un análisis de documentos. El investigador identifica, estudia y, luego, sintetiza los datos para proporcionar un conocimiento del concepto o del suceso pasado que puede o no haber sido directamente observable” (McMillan y Schumacher, 2011, pp. 47-48). De ahí que, la información consultada fueron investigaciones provenientes de fuentes primarias y secundarias, retomando aspectos como objetivos, metodología, hallazgos y resultados.

Al respecto, el balance analítico hace alusión a quince investigaciones, las cuales tienen articulación con objeto de estudio, es decir, con la lectura y la escritura. De manera que, comprendió cuatro etapas, la primera consistió en buscar investigaciones relacionadas a la lectura y la escritura; en la segunda, se seleccionaron aquellas que tuvieran una introducción, metodología, resultados o hallazgos y conclusiones; en la tercera, se agruparon de acuerdo al objeto de estudio, los sujetos de investigación o el contexto; y en la cuarta, se analizó cada una, tomando lo más relevante para la elaboración del artículo. 
Después de todo, los aportes develaron que la lectura y la escritura son procesos continuos que permite a los alumnos entender parte de una realidad. Asimismo, se recuperó que los actores educativos siguen interesados en la lectura y la escritura como habilidades mecánicas y no como prácticas sociales, en donde, lo importante sea la interacción, la comunicación y el reconocimiento de sí mismo y de los otros.

\section{Metodología}

Cabe señalar que el presente no fue únicamente una recopilación de investigaciones, sino que también correspondió a un análisis de las mismas, de ahí que, se tomaron producciones del contexto regional, nacional e internacional, procurando que dieran cuenta del objeto de estudio, en este caso la lectura y la escritura. Mismas que han sido entendidas "como prácticas sociales más que como un conjunto de destrezas que se centran en la manipulación mecánica de elementos aislados del texto" (Kalman, 2003, p. 43). De esa manera, tienen que ver con la comunicación, interacción y reconocimiento, en un contexto determinado.

En este sentido, para el desarrollo de la investigación, el método empleado fue cualitativo, con modalidad no interactiva, ya que, la revisión analítica consideró investigaciones relacionadas con la lectura y la escritura. En concreto, "los documentos autentificados son la fuente principal de datos. El investigador interpreta «hechos» para proporcionar explicaciones del pasado y descubre los significados educativos colectivos que pueden subyacer a prácticas y temas actuales" (McMillan y Schumacher, 2011, p. 48). Esto implicó que la técnica de recogida de información fueran repositorios digitales.

Asimismo, se consideraron cuatro etapas; la primera consistió identificar investigaciones relacionadas con la lectura y la escritura en el contexto de educación básica, tomando información de revistas especializadas como, Revista Iberoamericana de Educación, Revista Mexicana de Investigación Educativa, Perfiles Educativos; y tesis de maestría del Instituto Superior de Ciencias de la Educación del Estado de México, cuya publicación fuera entre el 2000 y 2018. Por lo tanto, en un inicio se identificaron treinta y cinco artículos digitales y diez tesis de maestría, observando que los repositorios mostraban un mundo de información, la cual se tenía que seleccionar minuciosamente. 
En la segunda etapa se seleccionaron las investigaciones, ocupando aquellas que se apegaron a la lectura y la escritura de alumnos en educación básica, por lo que se fueron dejando artículos y tesis, de modo que sólo se analizaron diez artículos y cinco tesis de maestría. De las cuales se retomó la estructura, es decir, que tuvieran introducción, metodología, resultados o hallazgos y conclusiones, para reunir lo más relevante.

En la tercera etapa, a medida que se revisaban los artículos y tesis de maestría, se determinaron otras características como, el contexto (preescolar y primaria), que la población no únicamente fueran los alumnos, también los docentes, descartando aquellas investigaciones relacionadas con sujetos de educación media superior y superior, ya que el interés se centraba en educación básica. Por lo tanto, se agruparon tratando de guardar similitud con el objeto de estudio, sujetos de investigación y/o contexto.

Así, la organización contempló seis apartados, siendo: la lectura y la escritura de alumnos en situación de vulnerabilidad; la lectura y la escritura en educación preescolar; la lectura y la escritura en educación primaria; las prácticas docentes con relación a la lectura y la escritura; las etapas de la lectoescritura; y, las prácticas de lectura y escritura en el ámbito familiar y sociocultural.

Finalmente, en la cuarta etapa, se extrajo la información considerada significativa, por consiguiente, se encontraron elementos que permitieron dar una idea de lo abordado en las investigaciones. En este tenor, fue evidente que la lectura y la escritura pocas veces la estudiaron de manera simultánea, llegando a pensar que para los investigadores resultó más interesante la lectura, siendo obvia la separación que hicieron entre una y otra.

\section{Resultados y discusión}

\section{La lectura y la escritura de alumnos en situación de vulnerabilidad}

Una de las principales tareas de la escuela es, incluir a todos los alumnos en el proceso de la lectura y la escritura, por ello los actores educativos se han ocupado de brindar herramientas necesarias para que la población vulnerable, en este caso los alumnos con discapacidad, logren las competencias establecidas en los Programas de Estudio. Por lo tanto, a raíz de lo que acontece en las escuelas y los salones de clases, los investigadores se han interesado por temas de esta índole. 
En este tenor, Ramos (2004), en su investigación titulada "Enseñar a leer a los alumnos con discapacidad intelectual: una reflexión sobre la práctica", enfatizó la importancia de la lectura en la escuela, convencido que no existe un método específico para enseñar a leer a los alumnos con discapacidad intelectual, se enfocó a la enseñanza de la lectura dirigida partiendo de una perspectiva teórico práctica.

Señaló que los alumnos no constituyen un grupo homogéneo, ya que posee sus propias características, necesidades e intereses, entonces, los docentes deberían analizar los métodos de lectura más favorables para cada situación, de acuerdo con esto "podríamos hablar de estrategias metodológicas que favorecen el aprendizaje lector de todos los alumnos, incluidos aquellos que tienen discapacidad intelectual" (Ramos, 2004, p. 203).

Por lo que concluye que, la lectura es un proceso largo y con algunas dificultades, y aún más para los alumnos que presentan discapacidad intelectual. Entonces, deben plantear varios caminos para llegar a ella, olvidando que solo existe un medio para aprender a leer; ya que, la situación de cada alumno es diferente, así como los procesos y etapas. Sin embargo, la escuela tiene otras exigencias, lo que pretende es que el alumno lea y escriba, sin importar los medios, ante esto los docentes utilizan diferentes métodos en el desarrollo de estas habilidades.

Por otra parte, en el artículo “Adquisición de la lectoescritura en niños sordos: Una visión desde los profesores de Chile", de Lissi, Grau, Raglianti, Salina y Torres (2001), pretendieron "presentar una visión actualizada del proceso enseñanza-aprendizaje de la lectoescritura en distintos establecimientos de la Región Metropolitana que atienden a niños sordos”, el estudio lo llevaron a través de grupos focales, con 40 profesores en una Región Metropolitana de Chile.

Indagaron la percepción de los profesores respecto a las dificultades que enfrentan los alumnos sordos en la adquisición de la lectoescritura. Para ello, realizaron encuentros con la participación de entre 6 y 12 profesores en cada escuela, entregando a estos una pauta, en donde anotaron sus comentarios y opiniones verbales durante la sesión; también, incluyeron conversaciones informales. En las pautas retomaron aspectos como,

Enfoque metodológico utilizado... proceso de enseñanza-aprendizaje de la lectoescritura, modalidades de comunicación utilizada, creencias y expectativas 
asociadas a la educación de niños sordos, dificultades percibidas en el proceso de adquisición de habilidades de lectoescritura... y percepción de la formación de profesores para realizar exitosamente su tarea. (Lissi, Grau, Raglianti, Salina y Torres, 2001, p. 43).

La información que obtuvieron fue analizada y encontraron resultados como, la identificación de creencias, dificultades, tensiones y problemas que viven los profesores en su interacción y trabajo con los alumnos sordos. Dentro de los hallazgos hubo la falta de consenso entre los profesores respecto a la metodología de enseñanza y comunicación, además, de una frustración sobre los logros obtenidos. Concluyen que, es necesaria la investigación en esta área, ya que, se requiere de enfoques y estrategias específicas para alumnos sordos.

\section{La lectura y la escritura en educación preescolar}

La investigación titulada "Las prácticas de lectura y escritura en tercer grado de preescolar" de Ayala, (2016) tuvo como objetivo "conocer las prácticas de la lectura y la escritura que realiza la docente de preescolar en una escuela de organización completa en un contexto urbano" (p. 29). Realizó el trabajo de campo con una maestra de preescolar quien tenía a su cargo el tercer grado, la razón por la cual la eligió fue "por tener conocimiento de que su práctica de lectura y escritura es exitosa, hasta el grado que sus niños egresan del preescolar con un conocimiento formal de estos aprendizajes" (p. 30).

Se enfocó en una investigación cualitativa, usando la etnografía, empleando instrumentos como el registro de observación, las fotografías, las guías de entrevista y las notas de campo. La investigación la sustenta con la teoría crítica, con apoyo de la sociología de la vida cotidiana; esta última le ayudó a comprender el actuar de los alumnos en su acontecer escolar, teoriza tres conceptos: práctica docente crítica, lectura y escritura, y educación preescolar.

Dentro del contexto contempló el internacional, nacional, estatal, local y escolar. La información la analizó, sistematizó e hizo una interpretación descriptiva llegando a concluir que existen muchos factores implicados para comprender cómo una docente lleva a cabo el proceso de aprendizaje en sus alumnos, uno de ello es que son reproductoras del conocimiento e imponen lo que se tienen que conocer y aprender. 
Consideró que lo más natural para lograr los aprendizajes de la lectura y la escritura sería "a través de las experiencias vividas, de poner al sujeto en situación de aprender, respetando la posibilidad y habilidad de cada niño y niña, haciendo de esto no un aprendizaje impuesto sino vivido, interiorizado y sobre todo disfrutado" (Ayala, p. 106).

En esta misma línea, Picazo (2016) realizó una investigación sobre "La lectura y escritura como práctica social, una posibilidad para favorecer las prácticas letradas en preescolar”, a través del desarrollo de un proyecto institucional, con la modalidad de talleres dirigidos a los alumnos y con un proceso de acompañamiento a la práctica docente. De manera que, contempló en la intervención pedagógica cuatro fases.

En la primera, realizó el diagnóstico de las prácticas de enseñanza de la lectura y la escritura, con una duración de cinco meses. Para esta fase trabajó en dos escuelas de nivel preescolar, identificando "que las docentes desarrollan prácticas de enseñanza tradicional basadas en la concepción de la tecnología educativa de los años 80’, fundada en el entrenamiento técnico o mecánico" (Picazo, 2016, p. 26).

En la segunda, estableció la propuesta de intervención de la enseñanza de la lengua escrita como práctica social, la cual apuntó a favorecer el acercamiento del alumno a la lengua escrita en un sentido natural y social, con una duración de nueve meses; la tercera fue el acompañamiento a la práctica docente a fin de conducir los procesos de la lectura y la escritura; y la cuarta, la evaluación basada en la pertinencia del proyecto taller, mediante las evidencias de trabajo fundadas en los logros alcanzados por los alumnos.

El trabajo de campo lo llevó en un Jardín de Niños, ubicado en la zona oriente del Estado de México en el municipio de Ixtapaluca, seleccionando como protagonistas a dos, de seis docentes y sus grupos. "Metodológicamente la investigación se sustenta mediante la etnografía, la intervención pedagógica y el análisis documental de los cuatro últimos programas educativos de nivel preescolar" (Picazo, 2016, p. 9).

Llegando a concluir que el Programa de Estudios 2011 por el simple hecho de ser un programa vigente no garantiza un tipo de enseñanza en correspondencia con el enfoque sociocultural, el cual suscribe a la lengua escrita en términos de práctica social, sino permanecía latente una enseñanza 
tradicional, es decir, que las prácticas docentes eran casi invariables y no experimentaban mejoras evidentes a través de los años, por lo que se iban sedimentando.

\section{La lectura y la escritura en educación primaria}

La lectura y la escritura se encuentran presentes en todos los contextos, los sujetos continuamente las usan para mantener comunicación con los demás, sin embargo, las prácticas convencionales tienen mayor predominio dentro del salón de clases, de esta manera Guevara, López, García, Delgado y Hermosillo (2008), realizaron una investigación sobre "Niveles de escritura en alumnos de primer grado, de estrato sociocultural bajo". Derivado de esto, el interés de los investigadores se centró en,

Dar cuenta del proceso de desarrollo y el nivel de eficiencia que logran en sus habilidades de escritura los alumnos de primer grado de primaria, pertenecientes a escuelas públicas mexicanas de nivel sociocultural bajo, describiendo el tipo de escritura que desarrollan en ese nivel educativo. (Guevara, López, García, Delgado y Hermosillo, 2008, p. 48).

Para llevar a cabo su investigación, primero seleccionaron la muestra, a través de la técnica de muestreo no probabilístico intencional, de tal manera que el total de estudiantes fue de 262, perteneciente a tres escuelas primarias públicas de la zona metropolitana del Estado de México, sin embargo, solo trabajaron con 165, por presentar características en común como, nivel socioeconómico, edad, grado escolar y antecedentes escolares.

Después, para evaluar las habilidades de copia, dictado, composición y comprensión en diferentes momentos del primer ciclo de educación formal utilizaron el Inventario de Ejecución Académica (IDEA) desarrollado por Macotela, Bermúdez y Castañeda (2003), en su parte correspondiente a la evaluación de escritura en primer grado de primaria. Con respecto a los resultados encontraron que los alumnos iniciaron con niveles preacadémicos y lingüísticos inferiores; observaron en la evaluación escrita, habilidades para copiado de palabras, no así para copiar enunciados y textos, en estos identificaron mayor problema en.

Uno de los hallazgos fue que "el nivel obtenido en las habilidades de escritura a lo largo del ciclo escolar guardó una estrecha relación con los niveles preacadémicos y linguísticos mostrados 
al ingresar al primer grado" (Guevara, López, García, Delgado y Hermosillo, 2008, p. 56). Subrayan que el nivel socioeconómico y cultural influyó en el desarrollo de algunas habilidades, por ejemplo, cuando la familia no estimula el lenguaje oral del alumno, hay mayor probabilidad de que presente dificultad para atender la palabras y sonidos.

Los mismos investigadores, Guevara, López, García, Delgado, Hermosillo y Rugerio, (2008), retoman la población de 165 alumnos, pero enfocándose a las "Habilidades de lectura en primer grado en alumnos de estrato sociocultural bajo", consideran que la problemática ha estado presente por varios años, y no solo en los aspectos de la lectura, sino también de la escritura.

Se inclinaron por identificar qué pasaba con las habilidades lectoras, de ahí que, aplicaron el Inventario de Ejecución Académica (IDEA), de Macotela, Bermúdez y Castañeda (2003), en la parte correspondiente a la lectura de primer grado. Con respecto a los resultados, identificaron que la mayoría de los alumnos no sabían leer y que al ingresan a primer grado de primaria pueden llegar o no con antecedentes de lectura, debido a características propias, además,

Para el final del ciclo escolar, apenas un poco más de la mitad de los participantes (54\%) logró leer correctamente las seis palabras de la prueba de lectura oral, menos de la mitad pudo leer los cuatro enunciados aislados y únicamente $29 \%$ fue capaz de leer los siete enunciados del texto. (Guevara, López, García, Delgado, Hermosillo y Rugerio, 2008, p. 591).

Concluyen que, los alumnos deben cumplir ciertas características para lograr los aprendizajes de primer grado, por ejemplo, ingresar a primaria con seis años cumplidos y cursar por lo menos dos años de preescolar. Al parecer estas condiciones favorecieron el proceso de lectura, sin embargo, pocos padres de familia tenían idea de ello, por lo que podían o no mandar a sus hijos al preescolar, lo que generó una desventaja con los alumnos que asistieron, al llevar conocimiento de lectura, mientras que otros mostraron pocas habilidades en ésta.

Por otra parte, la investigación titulada "La adquisición de la lectoescritura en educación básica desde las teorías interaccionistas sujeto-ambiente, el caso de tres escuelas primarias de Tuxtla Gutiérrez, Chiapas", de López, Ramos, y Mancilla, (2009), plantearon como objetivo "conocer el papel de la enseñanza en el desarrollo de las habilidades para el dominio del proceso lectoescritor", 
consideraron que existe innumerables textos que abordan la problemática de la lectura, pero no como la adquisición de la lectoescritura.

Derivado de ello, utilizaron como estrategia metodológica la evaluación de 77 alumnos del primer ciclo de educación primaria, quienes presentaban problemas en la adquisición de la lectoescritura, utilizando el instrumento BENHALE, de forma paralela observaron y entrevistaron a 12 profesores, desde la perspectiva etnográfica. En sus resultados identificaron que los alumnos evaluados mostraron lectura poco fluida y escritura con omisiones y sustituciones de letras, a causa del ausentismo o niveles de maduración inferior a la edad, al respecto refirieron que,

El 34\% tienen problemas con la coordinación visomotora, 74\% con la memoria motora, $54 \%$ con la percepción y discriminación visual, $72 \%$ con la articulación, $71 \%$ con el vocabulario; $11 \%$ con la estructuración espacio temporal; $16 \%$ con la percepción y discriminación auditiva, 50\% con la memoria visual inmediata y 36\% con la memoria auditiva-lógico inmediata. (López, Ramos, y Mancilla, 2009, p. 10).

En este sentido, identificaron comportamientos rutinarios entre los docentes, quienes hacían uso del libro de textos y alfabeto móvil, con poca atención a los alumnos con retraso en la lectoescritura e inclinación hacia los más aventajados, utilizando a estos como modelos. Asimismo, encontraron que los maestros justificaron que los problemas eran el resultado de situaciones externas, es decir, que los alumnos no aprendían a causa de antecedentes de nacimiento y del poco apoyo que recibían de sus padres.

Las investigadoras concluyen que los problemas detectados se encontraban en la habilidad motora, la articulación y el vocabulario, aunado al trabajo del maestro quien percibía a los alumnos de manera homogénea.

Autores como Smith, Jiménez y Ballesteros (2005) han estudiado el desarrollo de la lectoescritura en los contextos familiar y escolar; así, su objetivo fue "entender los procesos e ideologías que contribuyen a la formación de lectores y escritores en México"; propusieron que el hogar, la comunidad y la escuela son espacios donde se desarrolla la lectoescritura, porque allí se da la interacción con los demás. 
Retomaron los términos lectoescritura y alfabetización, también, "usan la expresión ecología de la lectoescritura a partir del reconocimiento de que las habilidades, creencias y prácticas desarrolladas en un ambiente o contexto no necesariamente son semejantes a las que se desarrollan en otros" (Smith, Jiménez y Ballesteros, 2005, p.15). La investigación fue de corte cualitativaetnográfica, trabajaron en tres primarias del Estado de Puebla, para ello contemplaron una privada, una semiprivada y otra pública.

Para la recolección de datos aplicaron la observación en el salón de clases; entrevistaron a los alumnos de primer y cuarto grado de primaria, a los profesores y directores, asimismo, realizaron un análisis de textos producidos por los alumnos. Con relación a los resultados mencionaron que, la escritura está controlada en los tres tipos de escuelas, ya que la producción escrita se basó en textos cortos y sencillos, a través del dictado y la copia; en lectura dedican poco tiempo, en ocasiones la sustituye con la ausencia del maestro o como una actividad fuera del currículo; mientras que la preocupación de los padres se centraba más en la escritura que en la lectura.

Dentro de los hallazgos, señalaron de manera tentativa que la ausencia de uno o más miembros de la familia, por tiempos prolongados, puede ser un factor negativo en el desempeño educativo de los alumnos y que los textos producidos en la escuela son muy diferentes a los producidos en la comunidad.

Finalmente, Flórez, Torrado, Mondragón y Pérez (2003), realizaron la investigación titulada "Explorando la metacognición: evidencias en actividades de lectura y escritura en niños y niñas de 5 a 10 años de edad", cuyo objetivo fue "describir y categorizar la operaciones metacognitivas evidenciadas en los procesos de lectura y escritura en niños y niñas que se encontraban cursando grado transición y básica primaria en una institución educativa perteneciente al estrato socioeconómico tres en la ciudad de Bogotá (Colombia)”.

Plantearon que la metacognición puesta en juego para la lectura se evidencia en la conciencia que tienen los alumnos sobre su propósito en la misma, de su comportamiento en la lectura y el uso apropiado de estrategias para remediar fallas. Por lo tanto, trabajaron con una población de 36 alumnos, pertenecientes a una institución educativa, en la ciudad de Bogotá, seleccionados con igual edad cronológica. 
Los instrumentos aplicados fueron dos, el primero llamado la Tarea de Yuche (TAY) el cual les permitió evidenciar operaciones metacognitivas en lectura, el otro fue la Tarea de Cox (TBC), cuyo objetivo era evidenciar operaciones metacognitivas en escritura. Así, los resultados indicaron que, en los desempeños metacognitivos de escritura, la mitad de la población se ubicó en el nivel I, aumentando el desempeño en comprensión lectora y producción escrita.

La otra parte de la población se ubicó en el nivel II y una pequeña proporción en el nivel más exigente, lo mismo ocurrió en los desempeños metacognitivos de lectura, evidenciándose que en los primeros niveles (I y II) se encuentran más alumnos y pocos alcanzan el nivel más exigente. Con respecto a los hallazgos, confirmaron que a mayor nivel de metacognición se es mejor lector y que existe una correspondencia entre comprensión lectora y producción escrita, además, reconocieron que el desempeño de los alumnos mejora a medida que avanzan de grado escolar.

\section{Las prácticas docentes con relación a la lectura y la escritura}

La lectura y la escritura han sido investigadas a partir de la producción de los alumnos, pero ¿qué sucede dentro de la práctica docente?, ¿qué pasa con la interacción maestro-alumno y la apropiación de la lectura y la escritura?, derivado de estos cuestionamientos, se presentan investigaciones relacionadas al respecto.

La investigación realizada por Alejandro (2007), tiene como título "La construcción cotidiana de las estrategias de enseñanza para la lectura y escritura en primer grado de educación primaria”, se enfocó al trabajo que realiza el docente al interior del aula, el cual ha sido matizado por ideas, creencias y teorías que finalmente aterrizan en la enseñanza. Por lo tanto, el propósito fue “documentar y analizar la construcción cotidiana de estrategias de enseñanza para la lectura y la escritura en el primer grado de educación primaria".

Dentro de la investigación se encontraron apartados como, la descripción del contexto social e institucional de las escuelas; las estrategias de convocatoria y/o motivación para la enseñanza de la lectura y escritura $1^{\mathrm{a}}$ parte, contemplando juego y cuento; las estrategias de la enseñanza para la lectura y escritura $2^{a}$ parte, aquí se enfocó a la lectura en voz alta, el dictado y la redacción; y finalmente las estrategias de evaluación, incluyendo la supervisión del trabajo en forma grupal. 
El trabajo de campo lo realizó en dos escuelas primarias ubicadas en municipios del Estado de México, pertenecientes a un contexto urbano, en turno matutino y vespertino, con maestras de 1er. grado de primaria. La investigación la sustentó con el enfoque cualitativo apoyándose de la etnografía, utilizó para la recogida de información observaciones, entrevistas no estructuradas a docentes y directivos, así como, pláticas informales.

Las conclusiones apuntaron a que la redacción es la estrategia más utilizadas en la lectura y la escritura, la cual es practicada por los docentes, además, encontró que mientras un docente trabajó con mayor dificultad y socialización la escritura de los alumnos, los otros trabajaron de manera sencilla; por consiguiente, las actividades realizadas por los alumnos en el logro de la lectura y la escritura cuentan con una evaluación informal y continua.

Por otra parte, Ruiz (2012) señala que es conveniente reconocer los límites y alcances metodológicos del Programa Nacional para el Fortalecimiento de la Lectura y la Escritura en Educación Básica (PRONALEES), en especial las prácticas de los docentes, así, en su investigación titulada "La enseñanza de la lectura y la escritura, cuatro casos de Ixtlahuaca", pretendió "identificar los métodos ajenos al programa PRONALEES que se utilizan en el primer grado de educación primaria para la enseñanza de la lectura y escritura”.

En consecuencia, la estructura de su investigación fue, en primer lugar, la importancia educativa y social del PRONALEES, iniciando con los antecedentes históricos del programa, los propósitos y contenidos de la lectura y la escritura que enmarca el currículum; en segundo lugar, el papel del alumno y del docente en el PRONALEES, estableciendo la función de cada uno, además, de precisar el aprendizaje desde el punto de vista psicológico y pedagógico; y finalmente, el proceso de enseñanza de los docentes, es decir, las prácticas pedagógicas que llevaron a cabo en el salón.

Para el trabajo de campo, consideró a cuatro grupos de primer grado, pertenecientes a dos escuelas primarias del municipio de Ixtlahuaca, Estado de México, una ubicada en el contexto rural y la otra en el urbano. Los instrumentos utilizados en la recolección de información fueron la entrevista, la observación, la técnica de diario de campo y uso de videograbadora, esta última como recurso tecnológico. 
Después de analizar la información concluyó que se siguen presentando prácticas tradicionales, basadas en la escritura de planas de letras, sílabas, palabras y enunciados, y que la lectura es adquirida a través de deletreo, contrario a la propuesta metodológica que sugiere el PRONALEES. Señaló que las docentes del contexto urbano, al contar con un grupo numeroso optaron por llevar a la práctica el método onomatopéyico, con la aplicación de planas y constante repetición de letras, sílabas, palabras y enunciados para que los alumnos aprendieran a leer y escribir.

Además, identificó que los padres de familia no intervienen en el proceso educativo y que las autoridades educativas, como directivos y supervisores, no cumplen con la función de orientar o sugerir cambios, por lo que caen en la simulación de trabajar con los programas actuales, de hecho, los docentes no asumen la encomienda social de formar lectores y productores de textos con un fin comunicativo.

Por su parte, Pantaleón (2007), llevó a cabo la investigación con un grupo de alumnos de primer grado de educación primaria en una localidad rural del Rincón de Piedra Grande en el municipio de Luvianos, Estado de México, para ello implementó un "taller de lectura y escritura, con el propósito de mejorar la inmadurez de alumnos y facilitando a la vez la apropiación de las habilidades básicas" (p. 9).

Derivado que, en el medio rural la adquisición de la lectura y la escritura se han visto afectadas por factores como, las responsabilidades que atribuyen los padres de familia a sus hijos, la escuela alejada del lugar donde viven, las prácticas rutinarias que llevan a cabo los alumnos en el campo, el exceso de tareas de los docentes, la situación económica, la poca oportunidad de que asistan a preescolar, entre otros. Por lo tanto, dentro de los objetivos estableció:

Conocer el proceso de enseñanza que desarrolla el docente y cómo se apropia de la lectoescritura el alumno de primer grado en el contexto rural; y plantear, aplicar, seguir y evaluar alternativas pedagógicas, didácticas y metodológicas para la apropiación de la lectoescritura de los niños de la escuela primaria en el medio rural. (Pantaleón, 2007, p. 24).

Sustentó la investigación con soportes constructivista, basado en la teoría psicogenética en la enseñanza de la lectoescritura y consideró que el método más apropiado era investigación-acción, 
ocupando como instrumentos en la recolección de información fotografías, grabaciones de audio, entrevista, documentos oficiales y personales, cuestionarios y diario de campo. Por otra parte, el taller lo organizó en dos partes; lectura y escritura, tomando en consideración "los materiales, el tiempo, la organización y la evaluación para cada actividad” (Pantaleón, 2007, p. 94).

En lo relativo a los resultados, encontró que los alumnos lograron un avance significativo durante la aplicación del taller y que las habilidades de lectoescritura fueron beneficiadas; señaló que es preciso analizar los primeros acercamientos de la escritura, porque en el medio rural la instrucción es distinta en comparación con el urbano. Por ejemplo, que los padres de familia muestran poca preocupación por la educación y cuando sus hijos llegan a la primaria la responsabilidad recae en los docentes.

\section{Etapas de la lectoescritura}

Sabemos que, la lectura y la escritura en la escuela se trabaja a la par, de hecho, no se adquieren por separado, en este sentido, Montealegre y Forero (2006) llevaron a cabo una investigación titulada "Desarrollo de la lectoescritura: adquisición y dominio", señalaron que el desarrollo de la lectoescritura implica etapas e intervención de diversos procesos cognitivos, también, conocimientos y experiencias previas. Por lo tanto, para guiar su trabajo tuvieron como objetivos:

1. Analizar el desarrollo de la lectoescritura desde una concepción cognitiva y genética. 2. Abordar aspectos teóricos e investigativos, sobre las construcciones conceptuales del niño en el proceso de adquisición y sobre las habilidades necesarias para alcanzar el dominio de la lectoescritura. 3. Señalar cuestiones prácticas a partir de los aspectos teóricos e investigativos. (Montealegre y Forero, 2006, p. 26).

Las autoras propusieron fases para la lectoescritura con ciertas características, así, en la fase de adquisición presentaron los diferentes niveles conceptuales que desarrolla el alumno, desde edades tempranas, en su intento por comprender el lenguaje escrito. Describieron el papel de los gestos, el garabato, el dibujo y el juego, como las primeras construcciones con propiedades simbólicas. Mientras que, en la fase de dominio, mostraron una serie de procesos (perceptivos, léxicos, sintácticos, semánticos) y estrategias (metacognitivas e inferenciales) que determinaban el nivel de literacidad o dominio del sistema de escritura. 
Después de su análisis con diferentes investigaciones y estudios teóricos, concluyeron que las aportaciones de los autores aclaran el proceso de la lectoescritura desde su adquisición hasta su dominio; que los resultados de los diversos trabajos deberían conducir a una concientización de la práctica educativa; y que es preciso conocer los factores personales, sociales y ambientales del alumno porque estos determinan su estructura mental y, por lo tanto, la apropiación del conocimiento.

\section{Las prácticas de lectura y escritura en el ámbito familiar y sociocultural}

No solo en la escuela el alumno se apropia de la lectura y la escritura, sino que el contexto familiar es el primer lugar en donde puede tener los primeros acercamientos, para precisar se encuentra la investigación de Calderón (2015), "Producción escrita y literacidad familiar", quien tuvo como objetivo "indagar en la manera en la que, estudiantes de entre 7 y 10 años, se apropian de las prácticas y experiencias de lectura y escritura en el hogar en su producción textual” (p. 259).

Consideró una metodología con paradigma cuantitativo y las estrategias de análisis discursivo para el procesamiento de datos, trabajó con una muestra de 107 alumnos quienes cursaron segundo y cuarto año básico, reclutados por muestra de conveniencia o no probabilística de una escuela ubicada en la sexta región de Chile.

Mientras que en la recolección de la información los alumnos respondieron a una actividad tipo cuestionario, con interrogantes encaminadas a la literacidad familiar y en donde el autor consideró "actividades de literacidad, recursos para el aprendizaje y relación con los pares o padres. Agregaron también preguntas relacionadas a las percepciones y actitudes sobre la lectura", (Calderón, 2015, p. 266).

Después del análisis de los datos, llegó a los siguientes resultados: la mayoría de los alumnos declaró poseer actitudes positivas en torno a la lectura, aunque, los de cuarto año mencionaron que no desarrollan actividades asociadas a la lectura todos los días, a pesar que perciben tener muchos libros en su casa, también, encontró que a mayor edad poseen preferencias por ésta.

Dentro de los alcances de la investigación corroboró que los alumnos están expuestos diariamente a distintos recursos de lectura y escritura como, avisos publicitarios, revistas, panfletos, textos en televisión, entre otros; por lo tanto, resulta difícil que desempeñen actividades asociadas 
a la lectura y la escritura con baja frecuencia. Cabe destacar que el trabajo dio cuenta de algunos problemas específicos de la escritura.

Por otra parte, la investigación realizada por Gamboa, Muñoz y Vargas (2016), titulada "Literacidad: nuevas posibilidades socioculturales y pedagógicas para la escuela", partieron de un enfoque sociocultural, por lo que la lectura y la escritura fueron repensadas desde el concepto de literacidad. De ahí que, presentaron una amplia visión de la literatura más relevante de dichos aspectos.

Asimismo, hicieron un recuento de los cambios conceptuales dados a la lectura y escritura, asociándose con el proceso de alfabetización, prácticas sociales, prácticas letradas y literacidad; sustentan que la literacidad,

Se relaciona con todo lo que se hace con los escritos en una comunidad determinada e implica entender los discursos como un reflejo de la visión, los valores, actitudes del orden social, es decir, desde lo sociocultural, abriendo caminos para la multiliteracidad, entendida como la lectura de muchos textos en breves y variados espacios. (Gamboa, Muñoz y Vargas, 2016, p. 56).

Por otro lado, las investigaciones que presentaron abordan enfoques metodológicos cuantitativos y cualitativos comprendidos en diversos contextos, en este sentido, los apartados de análisis fueron: la literacidad como posibilidad de la construcción del sujeto, literacidad en las prácticas escolares y literacidad y reconfiguración del escenario escolar.

Acorde con ello, concluyen que en las investigaciones "se evidencia la urgencia de repensar las prácticas letradas en el escenario escolar, ya que estas han evolucionado al mismo tiempo que la tecnología", (Gamboa, Muñoz y Vargas, 2016, p. 65). Por lo tanto, se requiere de una reconfiguración de los actores educativos y su papel como sujetos letrados, ya que la cultura escrita se sigue viendo a manera de técnica para el desarrollo de habilidades cognitivas.

\section{Conclusiones}

En los últimos tiempos, se han generado una amplia gama de investigaciones referentes a la lectura y la escritura, desde diferentes campos disciplinantes como la educación, la psicología, la 
antropología, la sociología, entre otros, posibilitando otras miradas teóricas y metodológicas, a través de las cuales sugieren cambios en las concepciones, prácticas y formas de abordarlas.

Con lo anterior el balance de los documentos citados, muestran una coincidencia al situar a la lectura y la escritura como habilidades que son adquiridas en la escuela, por lo que han sido estudiadas en dicho contexto, sin embargo, en los últimos años han tomado el contextos familiar y social, porque es allí donde el alumno tiene sus primeras experiencias con la lectura y la escritura, en este sentido, el término que proponen es literacidad.

También, las investigaciones evidencian la tendencia de estudiar por separado la lectura de la escritura, pocas hacen referencia a ambas, ante esta postura pensamos que se debe al conocimiento sobre el objeto de estudios, así, la preocupación radica en la lectura, quizás porque en el contexto escolar se habla más de habilidad o competencia lectora y no de representaciones escritas. Esto no quiere decir que estudios con respecto a la escritura no existan, por el contrario, han quedado implícitos dentro de la lectura.

El retomar como contexto el escolar, fue una forma de determinar el espacio desde donde se hace investigación, este sentido, los sujetos fueron alumnos de preescolar y primaria, y los docentes, ya que resultó más práctico entender la lectura y la escritura desde el contexto en el que se tiene relación. Con respecto a los alumnos con discapacidad, también han sido investigados en el contexto escolar, aunque los trabajos siguen siendo pocos, en comparación con otra población.

Con relación a la metodología los investigadores tomaron el paradigma cualitativo, con enfoque etnográfico, otros la estrategia del análisis discursivo, la investigación-acción y trabajo documental. Coincidiendo en los instrumentos para la recogida de información (observación, entrevista, etc.); cabe señalar que cuatro de las quince investigaciones utilizaron instrumentos estandarizados a fin de evaluar la lectura y la escritura en el alumno.

Por otra parte, lo frecuente en las investigaciones fue que no describieron las concepciones que tiene los alumnos y los docentes de lectura y la escritura, se han ocupado más por describir procesos y habilidades, por lo que aquí hay un espacio a trabajar, es decir, partir de las prácticas sociales de los actores educativos con respecto a la lectura y la escritura, para desentrañar y comprender la interacción, identidades y subjetividades que en ellas se desenvuelven. 
Finalmente, los hallazgos se relacionan, al tomar en cuenta que la lectura y la escritura tienen que ver con las condiciones propias de los alumnos y las prácticas de los docentes, viéndose enmarcadas en lo tradicional. Se concluye, entonces, que la lectura y la escritura como prácticas sociales se trasladan a la realidad de las escuelas, sin embargo, los estudios siguen una tradición orientada a investigar la producción del conocimiento y no a describir desde lo sociocultural.

\section{Referencias}

Alejandro, R. (2007). La construcción cotidiana de las estrategias de enseñanza para la lectura y escritura en primer grado de educación primaria (Tesis de maestría). ISCEEM. Toluca, Edo. Méx.

Ayala, G. (2016). Las prácticas de lectura y escritura en tercero de preescolar. (Tesis de maestría). ISCEEM. Toluca, Edo. Méx.

Calderón, L. (2015). Producción escrita y literacidad familiar. Literatura y lingüística, 32, 259282.

Flórez, R., Torrado, P., Mondragón, B. y Pérez, V. (2003). Explorando la metacognición: evidencia en actividades de lectura y escritura en niños y niñas de 5 a 10 años de edad. Revista Colombiana de Psicología, 12, 85-98.

Gamboa, S., Muñoz, G. y Vargas, M. (2016). Literacidad: nuevas posibilidades socioculturales y pedagógicas para la escuela. Revista Latinoamericana de Estudios Educativos (Colombia), 12(1), 53-70.

Guevara, B., López H., García V., Delgado S. y Hermosillo G. (2008). Nivel de escritura en alumnos de primer grado, de estrato sociocultural bajo. Perfiles Educativos, 30(121), 4162.

Guevara, B., López H., García V., Delgado S., Hermosillo G. y Rugerio. (2008). Habilidades de lectura en primer grado en alumnos de estrato sociocultural bajo. Revista Mexicana de Investigación Educativa, 13(37), 573-597. 
Kalman, J. (2003). El acceso a la cultura escrita: la participación social y la apropiación de conocimientos en eventos cotidianos de lectura y escritura. Revista Mexicana de investigación educativa, 8(17), 37-66. Recuperado de http://www.redalyc.org/articulo.oa?id=14001704

Lissi, M., Grau, V., Raglianti, M., Salina, M. y Torres, M. (2001). Adquisición de la lectoescritura en niños sordos: Una visión desde los profesores de Chile. Psykhe, 10(1), 35-48.

López, G., Ramos, C. y Mancilla, G. (septiembre, 2009). La adquisición de la lectoescritura en educación básica desde las teorías interaccionistas sujeto-ambiente, el caso de tres escuelas primarias de Tuxtla Gutiérrez, Chiapa. Trabajo presentado en el X Congreso Nacional de Investigación Educativa. Recuperado de http://www.comie.org.mx/congreso/memoriaelectronica/v10/pdf/area_tematica_01/ponen cias/1055-F.pdf

McMillan, J. y Schumacher, S. (2011). Investigación educativa. España: Pearson.

Montealegre, R. y Forero, L. (2006). Desarrollo de la lectoescritura: adquisición y dominio. Revista Acta Colombiana de Psicología, 9(1), 25-40.

Pantaleón, G. (2007). Elementos iniciales para la enseñanza de la lectura y escritura en el primer grado de educación primaria en el medio rural: una experiencia de investigación-acción. (Tesis de maestría). ISCEEM. Toluca, Edo. Méx.

Picazo, C. (2016). La lectura y escritura como práctica social, una posibilidad para favorecer las prácticas letradas en preescolar. (Tesis de maestría). ISCEEM. Toluca, Edo. Méx.

Ramos, S. (2004). Enseñar a leer a los alumnos con discapacidad intelectual: Una reflexión sobre la práctica. Revista Iberoamericana de Educación, 34, 201-216.

Ruiz, R. (2012). La enseñanza de la lectura y la escritura, cuatro casos de Ixtlahuaca. (Tesis de maestría). ISCEEM. Toluca, Edo. Méx. 
Smith, P., Jiménez, R. y Ballesteros P. (2005). ¿Hay una pedagogía nacional de la lectoescritura? Una mirada a la construcción social de lectores y escritores en México. Revista latinoamericana de lectura y escritura: Lectura y vida, 26(1), 14-24.

Zavala, V. (2009). La literacidad o lo que la gente hace con la lectura y la escritura. En Cassany, D. (Comp.), Para ser letrados. (pp. 23-35). Barcelona: Paidós. 